\title{
«Ja, ich bin ein Kämpfer»
}

\section{Daniel Lüthi}

Freier Journalist und Fotograf, Medientrainer, Bern

Ein Häuserblock an der Strasse, der Monte San Salvatore ist nur von hinten zu sehen. Nichts Repräsentatives also, keine Spur von Dorfkönig. Der Dottore erwartet einen bereits im Gang vor der Praxis - mit breitem Lachen auf einem Wahlplakat. "Man kann mehr machen», heisst es darauf. Mit diesem Slogan war der Arzt, der auch Politiker ist, erfolgreich: Erneut wurde er im April ins kantonale Parlament gewählt. Diesmal aber für eine andere Partei - eine typische Denti-Geschichte.

\section{Kampf gegen die Kassen}

«Die Frau kann einer wechseln hier im Tessin», kommentiert er mit seinem typischen Schmunzeln, «die
Partei nicht. Ich machte es trotzdem - und gewann.» Wichtiger Zusatz: «Meinen Ideen bin ich selbstverständlich treu geblieben.» Auf seine frühere Partei, die CVP, ist er gar nicht mehr gut zu sprechen. «Die hat den sozialen Geist verloren. Und diskutiert nur noch, statt auch mal zu entscheiden und sich für etwas wirklich einzusetzen. Ich suchte frischen Wind und Utopien.» Denti trat den Grünen bei und setzt sich jetzt dort für seine Kernthemen ein, die - natürlich - vor allem im Gesundheitsbereich angesiedelt sind. Vehement widersetzt er sich vor allem den Krankenkassen: «Die rauben uns aus.» Seit 1996 bezahlten die Tessinerinnen und Tessiner viel zu hohe Krankenkassenprämien, erläutert er. Eine Studie der kantonalen Ärztegesellschaft
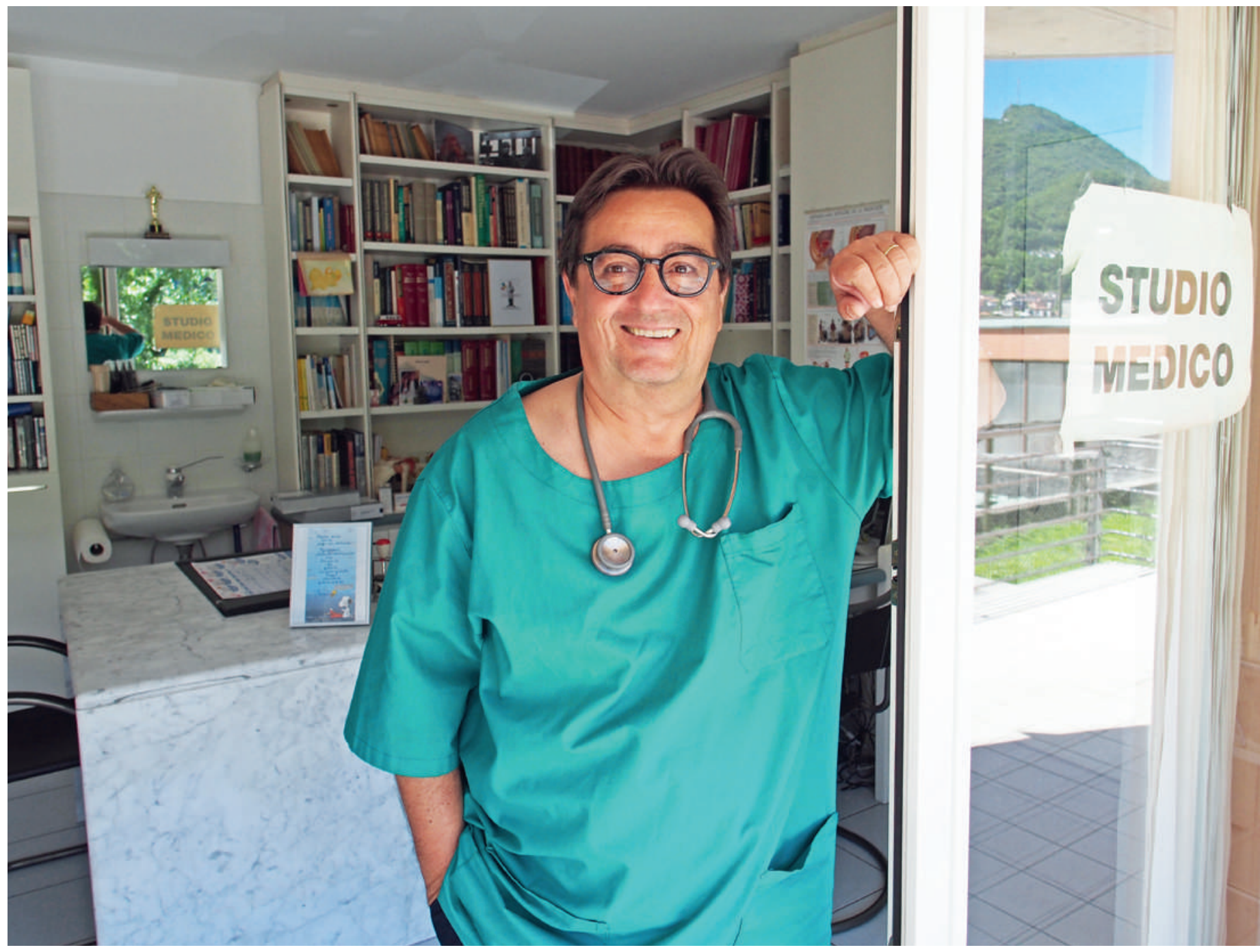
habe gezeigt, dass die Krankenkassen auf Kosten des Kantons Tessin Reserven von über 400 Millionen Franken angehäuft hätten. Dem gegenüber würden in anderen Kantonen die Prämien nicht einmal ausreichen, um die laufenden Kosten zu decken. «Es ist vordringlich, die Grundversicherung von den Zusatzversicherungen klar zu trennen", sagt Denti. Er wehrt sich also für Verschiedenes: höhere Taxpunktwerte und tiefere Prämien und damit für die Hausärzte und die Patienten, aber auch für den Kanton Tessin, der nicht Randregion sein soll.

Und auf einmal wird klar, dass sein Name Programm ist: «Denti» heisst «Zähne», und die zeigt er immer wieder gerne, nicht nur, wenn er lacht. «Wenn ich wütend bin, bin ich richtig wütend», sagt er mit eindringlichem Blick, «und dann habe ich auch Biss. Ja, ich bin ein Kämpfer.» Das begann bereits in der Jugend. "Ich bin Arbeitersohn, mein Vater starb früh. Und wer fand, so einer wie ich müsse nicht studieren, den habe ich eines Besseren belehrt.»

\section{Chef einer Zunft}

Wenn er so dasitzt und erzählt, wirkt er gemütlich und liebenswürdig, bestimmt aber sympathisch, keineswegs aggressiv oder verbissen. «Ein Geniesser bin ich auch», sagt er, «liebend gerne bin ich mit meinem Motorboot auf meinem Luganersee unterwegs oder mit unserem Irish Setter auf einem Spaziergang.» Zeit bleibt dafür wohl wenig. «Ich habe lange Tage - und arbeite gerne auch in der Nacht.»

Zur Illustration lässt er ein Gedicht von Alda Merini ausdrucken, das die Stille der nächtlichen Stunden preist. Schlafen? «Wenig.» Für alles reicht's nicht. Auf Kinder hätten er und seine Frau bewusst verzichtet, sagt er, Menschen ernst zu nehmen bedeute auch, Zeit zu haben für sie, im Beruflichen und im Privaten. So könne es schon vorkommen, dass Patienten lange auf ihn warten müssten. Dann aber widme er ihnen die nötige Zeit, und es mache ihm nichts aus, die Praxis erst am späten Abend zu verlassen.

Franco Denti hat neben seiner Praxis zahlreiche Ämter. So ist er Präsident der kantonalen Tessiner Ärztevereinigung, die hier eben nicht «associazione» oder ähnlich heisst, sondern "ordine», was eher eine Zunft ist - «eine stolze Zunft», wie Denti ergänzt. Das hat auch mit sozialem Status zu tun. «Ein Arzt sollte in einer Gemeinschaft doch eine ähnliche Stellung haben wie der Bürgermeister, der Pfarrer oder der Lehrer - früher zumindest.» Dabei meint er Anerkennung einer Autorität und Respekt, nicht Bewunderung oder Abgehobenheit.

«Ich bin nahe bei den Leuten», sagt er, «sie haben alle meine Handy- und meine Privatnummer, aber sie

\section{Franco Denti}

Dr. med. Franco Denti wurde 1957 in Sorengo bei Lugano geboren. Die Schulen absolvierte er in Lugano, das Medizinstudium in Pavia (Italien). Das italienische Staatsexamen machte er 1989. Dann bildete er sich weiter in verschiedenen Spitälern im Tessin, in Varese, Lausanne, Bern und Zürich,

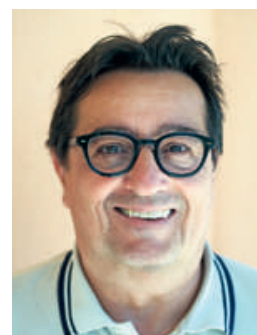
vor allem in Chirurgie, Interner Medizin, Kardiologie, Urologie und Gynäkologie. 1998 absolvierte er das Staatsexamen auch in Zürich, und er erhielt den Facharzttitel für Allgemeinmedizin. Im gleichen Jahr eröffnete er in Barbengo in der Nähe von Lugano eine Hausarztpraxis, nachdem es dort 60 Jahre lang keinen Arzt mehr gegeben hatte. Parallel dazu ist er Präsident der kantonalen Ärztevereinigung "Ordine dei Medici del Cantone Ticino", Delegierter der FMH, Vorstandsmitglied der SGAM und der Carnegie-Stiftung sowie Mitglied des kantonaIen Tessiner Parlaments. Franco Denti ist verheiratet. Er lebt mit seiner Frau in Lugano.

missbrauchen sie nicht. Gleichzeitig bringen sie mir Eier, Äpfel oder Wein, und sie laden mich ein zu einer Taufe oder Hochzeit.» Im langen Flur der Praxis hängen ganz selbstverständlich, wie in einer Kunstausstellung, die Pirelli-Kalender von 1998 bis heute, Fotografien mit Kultstatus eines Herstellers von Autoreifen Die halbnackten Damen würden ihr nichts ausmachen, sagt die Praxisassistentin, vielmehr habe auch sie Freude an diesem regelmässigen Geschenk eines $\mathrm{Pa}$ tienten.

\section{«Ich bin nahe bei den Leuten.»}

Einiges sei für einen Mediziner im Tessin wohl anders als nördlich der Alpen, ist Denti überzeugt, «die Beziehung zwischen Arzt und Patient zum Beispiel ist enger, persönlicher». Er braucht in diesem Zusammenhang den Begriff "Tessiner Medizinkultur», und erneut schwingt etwas Lokalpatriotismus mit. Auf dem Pult steht eine Fotografie, die den FC Malcantone Agno zeigt. «Nach dem Niedergang des FC Lugano wollte ich diese Mannschaft stärken, war deren Arzt.» In der Mitte steht der damalige Trainer der Lokalmatadoren, Vladimir Petković. Er trainiert heute die Schweizer Nationalmannschaft. "Man kann mehr machen.»

\section{Kritiker - auch intern}

Franco Denti engagiert sich vielerorts, auch ehrenamtlich. Seit rund 25 Jahren fährt er als Freiwilliger jeden August mit rund 200 Pilgern nach Lourdes. Oder er ist Vorstandsmitglied der Carnegie-Stiftung, die jedes Jahr Lebensretter auszeichnet und von Gutbetuchten 
grosszügig unterstützt wird. «Es ist schon recht, wenn Reiche Geld spenden», kommentiert er, «aber eigentlich hätten sie gar nie so reich werden dürfen, hätten ihr Geld vorher verteilen müssen.» Ein kritischer Geist, auch in den eigenen Reihen. So bekämpfte er die Managed-Care-Vorlage, die viele seiner Kolleginnen und Kollegen befürworteten. "Diese Vorlage war viel zu Krankenkassen-freundlich. Auch ich will Netzwerke, aber nach Kriterien, die der Ärzteschaft und den Patienten dienen, nicht den Kassen.»

Sogar eigene Netzwerke kritisiert Denti, so die SGAM, die im August mit der SGIM fusionieren will, oder die Vereinigung «Hausärzte Schweiz». «Das Tessin ist dort nicht vertreten, die wollen uns nicht. Und allzu oft sind sie zu Kompromissen auf zu tiefem Niveau bereit. Das bringt nichts.»

\section{«Man kann mehr machen.»}

Manchmal tönt beim Kämpfer Denti - wie jetzt wieder - auch Ernüchterung durch, Enttäuschung, ähnlich wie bei der politischen Partei, die er verlassen hat. Auf politischer Ebene habe er sich für bessere Rahmenbedingungen für die Hausärzte eingesetzt, sagt er. Einiges habe man da erreicht. "Aber jetzt, wo wir bessere Strukturen haben, fehlen uns immer mehr die guten Leute.» Es sind die bekannten Punkte, die auch ihn beschäftigen: «Die jungen Mediziner wollen weniger und geregelter arbeiten und mehr verdienen. Da haben wir ein Motivationsproblem.»

Gerade in Randregionen sei es deshalb schwierig, Nachfolger für Hausarztpraxen zu finden. Als er damals ziemlich zufällig nach Barbengo gekommen sei, habe er als Allgemeinmediziner in einem Dorf eine Praxis eröffnet, wo es vorher 60 Jahre lang keinen Arzt mehr gehabt habe. «Innerhalb von zwei Jahren erreichte ich die Ziele, die ich in meinem Businessplan nach fünf rund 5000 Patientendossiers, die lokale Bevölkerung komme zu ihm, im Sommer seien es auch viele Touristen, vor allem aus der Deutschschweiz und aus Deutschland. Hausbesuche gehören zur Tagesordnung.

\section{Alte Ziele, neue Plattformen}

Die Zukunft jedoch ist ungewiss. Zurzeit arbeitet ein italienischer Infektiologe in seiner Praxis mit. Für ihn eine Weiterbildung, ein Bekanntmachen mit den Verhältnissen in der Schweiz, für Denti mit all seinen Verpflichtungen eine willkommene Entlastung. Ob der Kollega aus Norditalien die Praxis dereinst übernehmen wird? Franco Denti zuckt mit den Schultern und äussert leise Hoffnung.

Wie auch immer: Denti wäre nicht Denti, würde er nicht für seine Anliegen immer wieder neue Plattformen ins Auge fassen. «Ich bin Pragmatiker, arbeite gerne an Projekten. Der Rahmen kann wechseln", sagt er. So ist er, nach dem Scheitern der Einheitskasse, unter anderem daran, die Idee einer interkantonalen Kasse weiterzutreiben. Bezogen auf seine politischen Mandate liegt sein nächstes Ziel in Bern, auf der nationalen Ebene. Bei den eidgenössischen Wahlen im Herbst wird er, der grüne Arzt und Politiker aus dem Südtessin, mit grösster Wahrscheinlichkeit für den Ständerat kandidieren.

Ganz nach seinem Motto: «Man kann mehr machen.» Oder, wie er zum Abschied sagt: «Wer etwas machen und bewegen will, findet immer einen Weg.»

\section{Die nächste Begegnung mit ...}

Am Ende jeden Monats stellt die Schweizerische Ärztezeitung eine Persönlichkeit vor, die sich im Gesundheitswesen engagiert. Im August schildert Daniel Lüthi seine Begegnung mit Pia Coppex, Pflegefachfrau und neue Präsidentin der Schweizerischen Gesellschaft für Biomedizinische Ethik. 